\title{
ÍNDICE DE MATERIAS Y AUTORES Volumen 6, números 1 al 4 del 2019
}

\section{Editoriales}

Diabetes, detección temprana para una enfermedad compleja

Luz Ángela Casas

Vol. 6, n. ${ }^{\circ} 1$, p. 4

La vitamina reina o la reina de las vitaminas

Ardila E

Vol. 6, n. ${ }^{\circ}$, p. 72

\section{El trabajo en equipo y la ACE}

Tovar $H$

Vol. 6, n. ${ }^{\circ}$ 3, p. 152

Editorial

Rojas $W$

Vol. 6 , n. ${ }^{\circ} 4$, p. 250

$15^{\circ}$ Congreso Colombiano de Endocrinología, Diabetes y Metabolismo

Marta Elena Marín Grisales

Vol. 6, supl. 1, p. 4

\section{Artículos originales}

Características clínicas, histopatológicas y terapéuticas del cáncer de tiroides en Colombia: serie de $\mathbf{1 . 0 9 6}$ pacientes

Edwin Antonio Wandurraga Sánchez, Lisseth Fernanda Marín Carrillo, Annie Katherine Natera Melo, Claudia Milena Gómez Giraldo, Freddy Niño Prato, Henry Mauricio Arenas Quintero, Rafael Castellanos Bueno, Néstor Alfonso López Pompey, Darío Arturo De La Portilla Maya, Karen Milena Fériz Bonelo, Alejandro Pinzón Tovar, Juan Pablo Dueñas Muñoz, Alin Abreu Lomba, Luis Felipe Fierro Maya, Juan Bernardo Pinzón Barco, José Luis Torres Grajales, Andrés Felipe Palacio Barrientos, Liseth Sánchez Orduz, Rafael Eduardo García Rey Vol. 6, n. ${ }^{\circ}$, p. 5-12

Caracterización cineantropométrica de la población que acude a la Clínica Las Américas. Estudio observacional, retrospectivo

Rosero RJ, Polanco JP, Jaramillo A, Cossio I, Gómez AM, Atehortúa AM, Loaiza S, Palacio JI

Vol. 6, n. ${ }^{\circ}$ 1, p. 13-21
Riesgo de diabetes y prediabetes en Manizales, estudio RIDIMA

Germán Camilo Giraldo González, Santiago José Morón Serrano, Daniel Fernando Giraldo Ceballos, María de los Ángeles Araque Coronado, Germán Camilo Duque Gallego, Nicolás Esteban Torres Riascos, Juanita González Escobar Vol. 6, n. ${ }^{\circ} 1$, p. 22-29

Caracterización de los hallazgos histopatológicos de tumores hipofisiarios y de la región selar en dos centros en Bucaramanga, Santander, entre 1992 y 2018 Juan Guillermo Sarmiento, María Paula Sarmiento, Laura Catalina Aguirre, Ludwing Joel Valero, Diana Katherine Saiz, Karoll Dayanna Aparicio, Jorge Luis Sanabria, Silvia Nathalia Gutiérrez, María Emma García, Isabel Cristina Bolívar Vol. 6, n. ${ }^{\circ} 1$, p. 30-34

Comparación del comportamiento glucémico de cuatro fórmulas nutricionales específicas para diabéticos mediante el uso de monitoreo continuo de glucosa Wandurraga EA, Marín LF, Galeano AM, Serrano-Gómez SE, Parra GA, Sarmiento JG

Vol. 6, n. ${ }^{\circ}$ 2, p. 74-79

Prevalencia de hipocalcemia postiroidectomía en cirugía de cáncer de tiroides

Mejía MG, Vega MP, Hakim JA

Vol. 6, n. ${ }^{\circ} 2$, p. 80-85

Factores de riesgo de Diabetes Mellitus tipo 2 en población adulta. Barranquilla, Colombia

Rodríguez M, Mendoza MD

Vol. 6, n. ${ }^{\circ} 2$, p. 86-91

Predictores de recaída en carcinoma diferenciado de tiroides en Colombia: estudio multicéntrico Marín LF, Torres JL, Wandurraga EA, Aristizábal C, Aristizábal N, Sylva DI, Ospina DC, Dueñas JP, Natera AK, Gómez CM,

Rondón MA, Serrano-Gómez SE

Vol. 6, n. $^{\circ} 2$, p. $92-100$

Eficacia de dos inhibidores de la fosfodiesterasa 5 combinados con ginkgo biloba en la disfunción eréctil en hombres con enfermedades crónicas

Espitia FJ, Orozco L

Vol. 6, n. ${ }^{\circ}$ 3, p. 155-163 
Expresión del receptor de andrógenos relativa al receptor de estrógenos (AR/ER) y su correlación con una señal génica de proliferación en cáncer de seno ER-positivo Rangel N, Aristizábal AF, Rondón M

Vol. 6, n. ${ }^{\circ}$ 3, p. $164-170$

Prevalencia del déficit de vitamina $D$ en mujeres posmenopáusicas de Armenia

Espitia FJ, Orozco L

Vol. 6, n. ${ }^{\circ}$ 3, p. 171-177

Variables hormonales y bioquímicas de la densidad mineral ósea y su correlación con hombres jóvenes obesos y no obesos sin diabetes

Romero MC, Sierra SF, Rincón JD, Martínez LA, Pulido JC, Maldonado LM, Franco R, Arteaga JM, Alzate JP, Camino JE Vol. 6, n. $^{\circ}$ 3, p. $178-187$

Histopatología testicular de ratas BIOU: Wistar expuestas a malatión

Serrano R, Hernández GA, Hung S, Lozano R, Paoli M, Gómez $R$ Vol. 6, n. ${ }^{\circ} 4$, p. $252-259$

Prevalencia y caracterización clínica del hipotiroidismo, en gestantes del Eje Cafetero (Colombia), 2014-2017 Espitia FJ, Orozco L

Vol. 6, n. ${ }^{\circ} 4$, p. $260-269$

Hipoglucemia en pacientes con diabetes mellitus en los servicios de urgencias de dos hospitales de Bogotá, 2015-2017

Chaves W, Martínez L, Díaz D, Hernández D, Sanabria M

Vol. 6, n. ${ }^{\circ} 4$, p. $270-277$

Ejercicio físico de alta intensidad como método de tratamiento para mejorar los niveles glucémicos en el paciente diabético

Pereira JE, Peñaranda DG, Pereira R, Pereira $P$, Mijangos $A D$

Vol. 6, n. ${ }^{\circ} 4$, p. $278-293$

\section{Artículos de revisión}

Tratamiento del hipoparatiroidismo con hormona paratiroidea

Esteban Cardona, Carlos Alfonso Builes-Barrera, Alejandro

Román-González

Vol. 6, n. ${ }^{\circ} 1$, p. 35-41

Hipocalemia refractaria como manifestación clínica de síndrome de Cushing ectópico: a propósito de dos casos y revisión de la literatura

Coronel Restrepo N, Mejía Zuluaga M, Aristizábal N, Torres

Grajales $J L$

Vol. 6, n. ${ }^{\circ} 1$, p. $42-50$
Acercamiento actualizado a la fisiopatología, clasificación y genética del síndrome de ovarios poliquísticos Orrego A

Vol. 6, n. ${ }^{\circ} 2$, p. 101-106

Gonadotropina coriónica humana, una hormona versátil y un marcador tumoral esencial en cáncer testicular de células germinales no seminomatosas

Marchán JC

Vol. 6, n. ${ }^{\circ}$ 2, p. $107-113$

Tuberculosis y vitamina D: una relación intrigante Ramírez-Ramos CF, Salamanca-Montilla JF, Correa $S$, Torres-Restrepo JM, Ramírez-Méndez DA, Rivera-Marín JD, Pinzón-Tovar A, Lastra-González G

Vol. 6, n. ${ }^{\circ}$ 2, p. $114-120$

Diagnóstico y manejo de la apoplejía hipofisaria Orrego A, Jácome $A$

Vol. 6, n. ${ }^{\circ}$ 3, p. $188-198$

Diabetes y pie diabético: una problemática mundial abordada desde la fisioterapia

Arroyo LD, Burbano JI

Vol. 6, n. ${ }^{\circ}$ 3, p. 199-209

Orbitopatía tiroidea: protocolo de manejo basado en revisión de la evidencia

Gómez C, Imitola A, Taboada LB, Henao DC, Marín LF, Camargo

J, Kattath L, Fonseca C, Colón C, Ascnher P

Vol. 6, n. ${ }^{\circ}$ 3, p. 210-217

Neuropatía autonómica cardíaca diabética

González J, Castaño O, Duque M

Vol. 6, n. ${ }^{\circ} 4$, p. 294-299

Osteomalacia: desde la sospecha clínica al tratamiento: revisión del tema

Duque V, Barrera N, Gómez J, Guerra V, Ceballos L, Aristizábal N Vol. 6, n. ${ }^{\circ} 4$, p. 300-307

Nuevos tratamientos médicos para el cáncer medular de tiroides

Román-González A, Mejía S, Zapata M

Vol. 6, n. ${ }^{\circ} 4$, p. 308-313

\section{Historia de la endocrinología}

Historia clínica de Henri de Toulouse-Lautrec

Enrique Ardila

Vol. 6, n. ${ }^{\circ} 1$, p. 51-54

http://revistaendocrino.org/ 
Percepción de las funciones testiculares no reproductivas antes de la testosterona: mitos y realidades

Jácome A

Vol. 6, n. ${ }^{\circ}$ 2, p. $133-140$

Descubrimiento de la testosterona

Jácome A

Vol. 6, n. ${ }^{\circ}$ 3, p. 231-235

Calciferoles: las hormonas del momento

Jácome A

Vol. 6, n. ${ }^{\circ} 4$, p. 320-327

\section{Páginas del residente}

Hiperglucemia inducida por glucocorticoides en el paciente hospitalizado: enfoque y manejo

Rossanna Tous Lopera, Andrés Felipe Coy Barrera, William Rojas García

Vol. 6, n. ${ }^{\circ} 1$, p. 55-61

Metabolismo óseo, osteoporosis y enfermedad inflamatoria intestinal en adultos

Duque JJ, Moreno DC, Arteaga JM, Franco R.

Vol. 6, n. ${ }^{\circ}$ 2, p. 127-132

Presentación atípica de una tiroiditis supurativa en una paciente inmunocompetente con compromiso vascular (síndrome de Lemierre)

Rojas W, Tovar H, Vargas JJ, Tous $R$

Vol. 6, n. ${ }^{\circ}$ 3, p. 218-227

Adenoma corticotropo silente: reporte de caso Gómez C, Taboada L

Vol. 6, n. ${ }^{\circ} 4$, p. 314-319

\section{Reportes de casos}

Hipertiroidismo secundario a coriocarcinoma metastásico, reporte de caso

Parra GA; Bolívar IC; Molina JF; García JA; Guevara H

Vol. 6, n. ${ }^{\circ}$ 2, p. 121-126

Variante C.113 C>T (P.SER 38PHE) de significado incierto asociada con caso típico de MEN1

Vargas $Y$.

Vol. 6, n. ${ }^{\circ}$ 3, p. 228-230

\section{Carta al editor}

Descubrimiento de la testosterona

Jácome A

Vol. 6, n. ${ }^{\circ} 4$, p. 328-329

\section{Obituario}

Doctor Rafael Gómez Cuevas

Jácome A

Vol. 6, n. ${ }^{\circ} 2$, p. 141

\section{5. ${ }^{\circ}$ CONGRESO COLOMBIANO DE ENDOCRINOLOGÍA, DIABETES Y METABOLISMO}

\author{
Resúmenes de las conferencias \\ Análogos del GLP-1 \\ Yadira Villalba Yabrudy \\ Vol. 6, supl. 1, p. 5 \\ Combinaciones de nuevos fármacos en el tratamiento de \\ la diabetes mellitus tipo 2 (DM2) \\ Sharona Azriel \\ Vol. 6, supl. 1, p. 5-6 \\ El perfil ambulatorio de glucosa: el nuevo estándar para \\ el manejo de la diabetes \\ Mahmood Kazemi \\ Vol. 6, supl. 1, p. 6-7
}

Implementación práctica de un modelo matemático en diabetes

Alex Ramírez Rincón

Vol. 6, supl. 1, p. 7-9

Inhibidores del cotransportador de sodio-glucosa tipo 2 (iSGLT2): ¿cambiaron las reglas del juego en diabetes? Orlando Carlos Castañeda López

Vol. 6, supl. 1, p. 9

Modelando la homeostasis de la glucosa desde la fenomenología

Carlos Esteban Builes Montaño

Vol. 6, supl. 1, p. 9-10

Disforias de género impuestas u obligadas: crónica (fábula y moraleja): entre la salud mental y el funcionamiento orgánico biogenético Silvia Cristina Chahín Ferreyra

Vol. 6, supl. 1, p. 11-12

Transición en la diabetes mellitus tipo 1 (DM1)

Juan José Ramírez Jiménez

Vol. 6, supl. 1, p. 13-14 
Hiperplasia congénita de suprarrenales

Mauricio Coll Barrios

Vol. 6, supl. 1, p. 14-15

Manejo del paciente con Klinefelter y Turner Martín Toro R.

Vol. 6, supl. 1, p. 16

Elección de la primera línea de tratamiento en tumores neuroendócrinos (TNE) enteropancreáticos de bajo grado en estado avanzado

Gloria Garavito

Vol. 6, supl. 1, p. 17

Enfoque terapéutico de adenomas no funcionantes (ANF) Karina Danilowicz

Vol. 6, supl. 1, p. 17-18

Hiperaldosteronismo primario

Carlos Alfonso Builes Barrera

Vol. 6, supl. 1, p. 18-19

Hipopituitarismo: causas y suplencia

Alejandro Pinzón Tovar

Vol. 6, supl. 1, p. 20-21

Insuficiencia adrenal primaria (IAP), diagnóstico y tratamiento

Doly Nubia Pantoja Guerrero

Vol. 6, supl. 1, p. 21-23

Metas en el manejo del paciente con acromegalia

Karina Danilowicz

Vol. 6, supl. 1, p. 23

Estrategias comportamentales para mejorar la adherencia al tratamiento a largo plazo de la obesidad Stella Guerrero Duque

Vol. 6, supl. 1, p. 24

Fisiología del colesterol y el papel de la PCSK9

Alejandro Román González

Vol. 6, supl. 1, p. 24-26

Orbitopatía tiroidea: conceptos básicos y diagnóstico clínico

Helena M. Coy Villamil

Vol. 6, supl. 1, p. 26-28

¿Cuándo un nódulo tiroideo es sospechoso?

John Jairo Duque Ossman

Vol. 6, supl. 1, p. 29-30
Estratificación dinámica del riesgo en el cáncer de tiroides

Natalia Aristizábal Henao

Vol. 6, supl. 1, p. 30-31

Factores de riesgo de recaída en el cáncer diferenciado de tiroides

Gloria Garavito

Vol. 6, supl. 1, p. 32

Orbitopatía tiroidea: herramientas e imágenes en el diagnóstico

Helena M. Coy Villamil

Vol. 6, supl. 1, p. 32-33

Hipotiroidismo en el embarazo: la hormona estimulante de la tiroides (TSH) en el diagnóstico y el tratamiento Natalia Aristizábal Henao

Vol. 6, supl. 1, p. 33-34

Hipotiroidismo subclínico (HSC): nuevos estudios, viejas advertencias

Harold H. García Touchíe

Vol. 6, supl. 1, p. 35-36

Inhibidores de la tirosina cinasa en el cáncer de tiroides Alejandro Román González

Vol. 6, supl. 1, p. 36-37

SPECT/CT en el cáncer de tiroides

Amelia de los Reyes

Vol. 6, supl. 1, p. 38

Impacto tiroideo de la disrupción endocrina

Amanda Páez Talero

Vol. 6, supl. 1, p. 39-40

Disruptores endocrinos y cerebro ¿hay alguna asociación patológica?

Maritza Pérez Mayorga

Vol. 6, supl. 1, p. 40-41

Endocrinología del estrés

Manuel Alberto Pérez Mazorra

Vol. 6, supl. 1, p. 42-43

La microbiota en la endocrinología

Sandra Mora Thiriez

Vol. 6, supl. 1, p. 43-44

Proceso de disruptores: del ambiente al epigenoma Fernando Lizcano

Vol. 6, supl. 1, p. 44-45 
Riesgo de cáncer de mama y de trombosis venosa profunda con la terapia de reemplazo hormonal (TRH)

Alejandro Pinzón Tovar

Vol. 6, supl. 1, p. 46-47

Uso de testosterona en el hipogonadismo masculino Leyda Yormary Martínez

Vol. 6, supl. 1, p. 47-48

\section{Trabajos de investigación}

Diabetes

Presentación oral

Aplicación de técnicas de inteligencia artificial para la estimación de insulina basal en pacientes con bomba de insulina

Seudónimo: Redes en Diabetes

Vol. 6, supl. 1, p. 49

Presentación oral

Asociación entre la obesidad y la depresión en pacientes con diabetes mellitus tipo 2 (DM2)

Seudónimo: Juan Pablo

Vol. 6, supl. 1, p. 50

Presentación oral

Control glucémico e hipoglucemia en pacientes tratados con terapia de bomba de infusión de insulina: estudio observacional

Seudónimo: Cabomba

Vol. 6, supl. 1, p. 50-51

Presentación oral

Factores de riesgo asociados a la diabetes de novo postrasplante entre los trasplantados renales de una institución de alta complejidad en Colombia entre 1995-2014

Seudónimo: Angelamvc

Vol. 6, supl. 1, p. 51-52

Presentación oral

Nivel de autocuidado y riesgo de pie diabético en usuarios de un servicio de salud del municipio de Palmira en 2018

Seudónimo: Burbano, J. I.

Vol. 6, supl. 1, p. 52-54

Presentación oral

Prescripción de inhibidores de la dipeptidil

peptidasa-4 (DPP-4): indicación e impacto en la hemoglobina glucosilada en un hospital de primer nivel de Colombia

Seudónimo: Achiote

Vol. 6, supl. 1, p. 55

Presentación oral

Prevalencia de los factores de riesgo asociados a la diabetes mellitus tipo 2 (DM2) en Colombia: análisis secundario del estudio PURE

Seudónimo: Apollonius de Memphis

Vol. 6, supl. 1, p. 55-56

Presentación oral

Prevalencia de lipodistrofia en adultos colombianos con diabetes mellitus tipo 1 y 2 (DM1 y 2)

Seudónimo: Madega

Vol. 6, supl. 1, p. 56

Presentación oral

Prevención de eventos cardiovasculares

en la población colombiana con diabetes mellitus

tipo 2 (DM2) durante el último trimestre de 2008 y 2018

Seudónimo: Saturno

Vol. 6, supl. 1, p. 57

Póster

Adulto mayor con diabetes y caídas a repetición:

factores asociados, análisis secundario del estudio SABE Colombia

Morros E, Venegas LC, Vargas MP, Chacón E, Chavarro D, Cano CA

Vol. 6, supl. 1, p. 67

Póster

Análisis comparativo entre pruebas clínicas y pruebas electrofisiológicas para la detección de neuropatía diabética en pacientes del servicio electrodiagnóstico de la Clínica Somefyr en 2018

Pineda LO, Peña AA, Monroy MA

Vol. 6, supl. 1, p. 67-68

Póster

Conocimiento, tratamiento y control de la diabetes mellitus tipo 2 en colombianos de 35-70 años: estudio PURE

García LM, Alvernia MJ, Camacho PA, Uribe S, Pérez M, López Jaramillo P

Vol. 6, supl. 1, p. 68

Póster

Consumo de hipoglucemiantes en pacientes con diabetes mellitus tipo 2 en la costa atlántica colombiana

Fernández JC, Orjuela LT, Ospina JD, Amézquita MA

Vol. 6, supl. 1, p. 68 


\section{Póster}

Determinantes de la prevalencia de prediabetes y diabetes mellitus tipo 2 en Colombia: estudio PURE Alvernia MJ, García M, Camacho PA, Uribe S, Pérez M,

López Jaramillo $P$

Vol. 6, supl. 1, p. 69

\section{Póster}

Dulaglutide Has Higher Adherence and Persistence Than Liraglutide and Exenatide Once-Weekly: 1-Year Follow-Up from United States Real-World Data

Landó LF, Mody R, Huang Q, Yu M, Zhao R, Patel H, Grabner M, Florez $S$ (Non-author Presenter)

Vol. 6, supl. 1, p. 69

Póster

Efecto hipoglucemiante de Anacardium occidentale $L$ como posible tratamiento en pacientes con diabetes mellitus

Parga CH, Tapia AD, Botero AC, Brito JE, De las Salas M, Fruto AC, Padilla J, Perea SS, Rosado CJ, Santodomingo NE, Calonge CD

Vol. 6, supl. 1, p. 70

Póster

Efecto de una intervención educativa en el conocimiento de la enfermedad del paciente con diabetes mellitus hospitalizado

Rojas N, Saldarriaga M, Builes CE, Arango CM, Prieto C, Jaramillo $C$

Vol. 6, supl. 1, p. 70

\section{Póster}

Gestión individualizada del riesgo en población diabética de Javesalud: experiencia de atención centrada en la persona en cuidado ambulatorio

Gordon SG, Páez PL, Castro DF, Robayo CX

Vol. 6, supl. 1, p. 70-71

\section{Póster}

Hiperglucemia severa de difícil manejo: síndrome de resistencia a los análogos de insulina

Pino JJ, Monsalve C, Aristizábal N

Vol. 6, supl. 1, p. 71

Póster

Lesser Estimated Glomerular Filtration Rate Decline with Dulaglutide Regardless of Weight Changes in People with Type 2 Diabetes and Moderate to Severe Chronic Kidney Disease

Tuttle KR, Lakshmanan MC, Rayner B, Busch RS, Zimmermann $A G$, Woodward BD, Botros FT, Florez $S$ (Non-author Presenter) Vol. 6, supl. 1, p. 71-72
Póster

Modelo matemático de la homeostasis de la glucosa: una mirada al comportamiento de pacientes con diabetes tipo 1

Ruiz N, Colorado V, Lema L, Builes CE

Vol. 6, supl. 1, p. 72

Póster

Modelo semifísico de base fenomenológica del rol del páncreas en la homeostasis de la glucosa en humanos Dávila MA, Tutalcha HA, Lema L, Builes CE

Vol. 6, supl. 1, p. 72

Póster

Nivel de adherencia a los tratamientos no farmacológicos de pacientes con diabetes mellitus, Barranquilla, 2017

Amador E, Montealegre L, Arteta M, De Oro C, Navarro G Vol. 6, supl. 1, p. 73

\section{Póster}

Niveles de vitamina $B_{12}$ en pacientes con diabetes mellitus en tratamiento con metformina Gómez JM, Laverde LA, González S, Penagos K, Rincón P, Tafurt Y Vol. 6, supl. 1, p. 73

Póster

Prediabetes, una condición frecuente en el ámbito hospitalario

Mejía HJ, Figueroa CL, Rodríguez RM, Romero DG, Gualdrón V, Sánchez $D$, Woolgrove $M$

Vol. 6, supl. 1, p. 73-74

Póster

Registro colombiano de pacientes con diabetes tipo 2 Reynales H, Castaño DM

Vol. 6, supl. 1, p. 74

Póster

Relación entre la severidad del síndrome de apnea-hipopnea del sueño (SAHOS) y la diabetes mellitus tipo 2 (DM2)

Mendoza T, Parra LA

Vol. 6, supl. 1, p. 74-75

\section{Póster}

Resultados en hospitalización y mortalidad por causa médica de la adherencia al tratamiento farmacológico en pacientes con diabetes mellitus tipo 2

Guzmán G, Iriarte MB, Ortega S, Ortiz D, Pardo N, Plaza E,

Tavares A, Martínez $V$

Vol. 6, supl. 1, p. 75 
Póster

Trasplante único de páncreas para paciente diabética tipo 1: reporte de un caso

Guzmán GE, Martínez V, Durán CE, Manzi E, Mesa L,

Schweineberg J, Posada JG, Villegas JI, Caicedo LA

Vol. 6, supl. 1, p. 75-76

Póster

Uso de agonistas del receptor de GLP1 para el tratamiento de la diabetes mellitus: experiencia de la vida real

Builes CE, Rojas N, Prieto C, Arango CM, Gutiérrez J,

Saldarriaga $M$, Granados EJ

Vol. 6, supl. 1, p. 76

\section{Trabajos de investigación} Endocrinología pediátrica

\section{Póster}

Determinación de los factores de riesgo en niños y jóvenes de la institución educativa Gimnasio Campestre Marie Curie para el desarrollo de dislipidemias, Bogotá D. C., 2018

Moscoso JM, Madrigal LJ

Vol. 6, supl. 1, p. 76-77

Póster

Espectro clínico de una serie de prolactinomas en pediatría

Suárez DV, Guzmán EC, Jaramillo JA, Lopera MV, Toro M

Vol. 6, supl. 1, p. 77

Póster

Estado nutricional y riesgo en el metabolismo óseo de niñas escolares en Pasto

Ramírez Prada D, Zafrilla P, Granja MA, Arévalo AC, Cerdá B

Vol. 6, supl. 1, p. 77-78

\section{Póster}

Síndrome metabólico y sus componentes individuales, según el sexo y criterios de definición en una cohorte de niños y adolescentes de Bucaramanga

Serrano N, Villa-Roel C, Gamboa-Delgado EM, Quintero-Lesmes DC Vol. 6, supl. 1, p. 78

Póster

Valoración de estilos de vida saludable de una población escolar de estrato medio en la ciudad de Bogotá D. C.

Cajiao V, Rodríguez S, Castro LP, Prieto C, Munar L, Aivasovsky I, Celis LG, Russi A

Vol. 6, supl. 1, p. 78-79
Trabajos de investigación Hipófisis y suprarrenales

Póster

52 semanas de tratamiento con análogos de somatostatina: respuesta bioquímica y tamaño tumoral en pacientes con acromegalia

Abreu A, Osorio CV, Salgado CA, Ramírez A, Barakat S, Carvajal $R$ Vol. 6, supl. 1, p. 79

Póster

Carcinoma adrenocortical con ACTH elevada: reporte de caso

Fierro F, Fuentes $O$, Coy $A F$

Vol. 6, supl. 1, p. 79-80

Póster

Diabetes no controlada, Cushing incidental. Reporte de caso

Jaramillo A, Mejía $H$

Vol. 6, supl. 1, p. 80

Póster

Fiebre de origen desconocido (FOD) secundaria a feocromocitoma

González AM, Fierro LF, Muñoz JD, Bermúdez LN, Guzmán JD, Vanegas JJ

Vol. 6, supl. 1, p. 80-81

Póster

Hallazgos de patología suprarrenal en autopsias en el departamento de patología del Hospital Universitario de Santander, 2012-2018

Mantilla AF, Lozano JF, Padilla LE, Mantilla JC

Vol. 6, supl. 1, p. 81

Póster

Hipofisitis por IgG4: reporte de un caso

Guzmán G, Hormaza A, Martínez V, Ortega S, Ortiz D

Vol. 6, supl. 1, p. 81

Póster

Hiponatremia por insuficiencia suprarrenal secundaria. Una serie de casos de un hospital universitario de tercer nivel en Pereira, Colombia

Guzmán ES, Giraldo J, Medina DA, Forero JE, Alzate JA, Vallejo S Vol. 6, supl. 1, p. 82

Póster

Linfoma de células B grande difuso que se presentó con infiltración hipofisaria, hipopituitarismo y compromiso del nervio oculomotor. Reporte de caso

Arias D, Revelo $S$, Forero JE, Vallejo $S$

Vol. 6, supl. 1, p. 82 


\section{Póster}

Supervivencia de pacientes con tumores

neuroendocrinos en un hospital de tercer nivel,

2012-2017

Pinzón A, Jiménez CE, Mondragón AE, Gutiérrez Y, García HA

Vol. 6, supl. 1, p. 83

\section{Póster}

Pegvisomant, un último recurso en acromegalia no respondedora al manejo medicoquirúrgico inicial: reporte de caso

Sánchez PE, Rojas W, Tovar $H$

Vol. 6, supl. 1, p. 83

\section{Póster}

Prolactinoma abscedado: absceso hipofisario asociado a un macroadenoma productor de prolactina. Importancia clínica y diagnóstica de las secuencias de resonancia en lesiones selares. Reporte de caso

Torres JL, Vargas MA, Coronel N, Aristizábal N

Vol. 6, supl. 1, p. 83-84

Póster

Síndrome de Cushing de difícil diagnóstico secundario al uso de un "producto naturista" para los dolores articulares y musculares

Fierro LF, González AM, Álvarez MA, Moreno GA, Rodríguez $S$, Tarquino $D$

Vol. 6, supl. 1, p. 84

\section{Póster}

Síndrome de Cushing, comportamiento posterior al tratamiento

Salgado CA, Muñoz JP, Bedoya V, Osorio CV, Tabares AA, Abreu A Vol. 6, supl. 1, p. 84-85

\section{Trabajos de investigación} Metabolismo óseo

Presentación oral

Niveles de 25-hidroxivitamina D en una población estudiantil universitaria de Armenia, Colombia Seudónimo: LJPV, DCG, OANC

Vol. 6, supl. 1, p. 57-58

Presentación oral

Niveles de vitamina D séricos y en lavado broncoalveolar en pacientes con sospecha de tuberculosis pulmonar Seudónimo: Vitaminadbal

Vol. 6, supl. 1, p. 58-59
Póster

Bajos niveles de vitamina $\mathrm{D}$ y su relación con la alteración de lípidos sanguíneos en mujeres posmenopáusicas de 2 municipios del departamento del Atlántico

Becerra JE, Rebolledo RC, Pabón AC, Suárez M, Sarmiento LA Vol. 6, supl. 1, p. 85

Póster

Déficit de vitamina D en hombres y mujeres con deseo sexual hipoactivo. Estudio de prevalencia

Espitia F, Orozco L

Vol. 6, supl. 1, p. 85-86

Póster

Determinación del consumo diario de calcio en la población colombiana

Arenas HM, Arias D, López VJ, Arias DR

Vol. 6, supl. 1, p. 86

\section{Póster}

Efficacy of Teriparatide Compared with Risedronate on Frax ${ }^{\circledR}$-Defined Major Osteoporotic Fractures: a Post-Hoc Analysis of the VERO Clinical Trial

Body JJ, Marin F, Geusens P, Zerbini C, Fahrleitner-Pammer A, Moericke R, Casado E, Stepan JJ, Minisola S, Lespessailles E, López-Romero P, Kendler DL, Florez S (Presenter Only)

Vol. 6, supl. 1, p. 86-87

Póster

Osteoporosis y baja masa ósea en mediciones de densitometría en Villavicencio, Meta, durante el 2018 Rosero FO, Garcés H, Beltrán A, Guerrero JF, Escobar LM, Galeano D, Karanauskas $S$

Vol. 6, supl. 1, p. 87

Póster

Osteoporosis, sarcopenia y correlación entre SARC-F y masa muscular por DXA en población colombiana: estudio piloto

Castrillón MP, Flórez AM, Vergara JE, Roa A, Quintero J, Parra G, Garay J, Cadena $M$

Vol. 6, supl. 1, p. 87-88

\section{Póster}

Patient Characteristics and Fracture Outcomes in Patients Previously Treated with Bisphosphonates or Treatment-Naïve in The Teriparatide Versus Risedronate VERO Clinical Trial

Hadji P, Marin F, Kendler DL, Geusens P, Russo L, MaloufJ, Lakatos P, Minisola S, López-Romero P, Fahrleitner-Pammer A, Florez S (Presenter Only)

Vol. 6, supl. 1, p. 88 
Póster

Prevalencia del déficit de vitamina $\mathrm{D}$ y factores de riesgo asociados en gestantes del Quindío

Espitia F, Orozco L

Vol. 6, supl. 1, p. 89

Póster

Prevalencia del déficit de vitamina $D$ en mujeres

posmenopáusicas de Armenia

Espitia F, Orozco Santiago L

Vol. 6, supl. 1, p. 89

Póster

Relación entre el índice TG/HDL-C, la resistencia a la insulina y la vitamina D sérica en mujeres posmenopáusicas del departamento del Atlántico, Colombia

Sarmiento L, Becerra JE, Rebolledo R, Suárez M, Barragán M, Sánchez $L$

Vol. 6, supl. 1, p. 89-90

Póster

Tabaquismo y vitamina D: una vía a la fragilidad

Pérez MU, Patiño D, Borda MG

Vol. 6, supl. 1, p. 90

\section{Trabajos de investigación} Obesidad y lípidos

Presentación oral

Análisis del contenido graso intrahepático por absorciometría de energía dual de rayos X (DXA) y su relación con los hallazgos ecográficos

Seudónimo: DXA-hepato

Vol. 6, supl. 1, p. 59

Presentación oral

Efectos de la obesidad materna sobre las características morfométricas de la placenta y el peso neonatal.

Prueba piloto

Seudónimo: Yhomar 2019

Vol. 6, supl. 1, p. 60

Presentación oral

Frecuencia del síndrome metabólico y el riesgo cardiovascular en pacientes con psoriasis en un centro de alta complejidad (noviembre 2017-mayo 2018)

Seudónimo: PSORIASIS-RCV-SM

Vol. 6, supl. 1, p. 60-61
Presentación oral

La relación de obesidad y baja fuerza de agarre u obesidad sarcopénica como predictor de la presencia de diabetes mellitus 2 (DM2)

Seudónimo: Diógenes de Sinope

Vol. 6, supl. 1, p. 61

Póster

Asociación entre algunas alteraciones metabólicas y la mala calidad seminal en hombres sobrepeso y obesos infértiles

Carmenate JV, Cabrera E, Monteagudo G, González N, González

$R$, Vicens $C$, Charara M, González P, Rodríguez L

Vol. 6, supl. 1, p. 91

Póster

Caracterización de pacientes y resultados de la cirugía bariátrica como tratamiento de obesidad en el Centro Médico Imbanaco, Cali, Colombia, de octubre de 2014 hasta febrero de 2019

Abella LM, Sterling DK, García AF, Santrich M, Arias $R H$, Vélez JP, Abreu A

Vol. 6, supl. 1, p. 91-92

\section{Póster}

Descripción del comportamiento del peso de un grupo de mujeres con cáncer de mama después del tratamiento Herrera GA, Insuasty JS, Rodríguez R, Reyes D, Rueda J,

Martínez NA

Vol. 6, supl. 1, p. 92

\section{Póster}

Efecto de la dieta intermitente frente a la continua en sobrepeso/obesidad en adultos: una revisión sistemática exploratoria y metaanálisis

Miranda $P$

Vol. 6, supl. 1, p. 92

Póster

Estrategias legislativas para la prevención de la obesidad en la población adulta colombiana: propuesta de mejoramiento

Cajiao V, Rodríguez S, Dávila V, Castro LP, Celis LG, Castiblanco HE, Russi A

Vol. 6, supl. 1, p. 92-93

Póster

Evaluación de factores de riesgo cardiovascular en mujeres de 12 a 25 años en Bogotá D. C., Colombia, para la implementación de programas de prevención primaria Moscoso JM, Camelo AM

Vol. 6, supl. 1, p. 93 


\section{Póster}

Frecuencia del síndrome metabólico en mujeres

posmenopáusicas de cuatro municipios del

departamento del Atlántico, Colombia

Rebolledo R, Ortega N, Becerra J, Sarmiento L

Vol. 6, supl. 1, p. 93-94

Póster

Frecuencia de sobrepeso y obesidad en mujeres posmenopáusicas del municipio de Santo Tomás, Atlántico, y su relación con el perfil lipídico Becerra JE, Sarmiento LA, Sánchez LP, Figueroa DA, Rebolledo RC Vol. 6, supl. 1, p. 94

Póster

Liraglutida como manejo de pacientes con pérdida de peso inadecuada posterior a manga gástrica Sterling DK, Abella LM, García AF, Santrich M, Abreu A

Vol. 6, supl. 1, p. 94-95

\section{Póster}

Liraglutida como tratamiento farmacológico para perder peso en pacientes poscirugía bariátrica

Daguer SA, Rojas EY

Vol. 6, supl. 1, p. 95

\section{Póster}

Nivel de sobrepeso/obesidad en estudiantes de medicina con sobrepeso/obesidad y su relación con el nivel de actividad física

Prado DG, Tabares SA, Messa MC, Revelo FJ, Murcia A, López AL Vol. 6, supl. 1, p. 95

\section{Trabajos de investigación}

\section{Tiroides}

Presentación oral

Cambios en el perfil tiroideo de la gestante obesa

Seudónimo: Linis

Vol. 6, supl. 1, p. 62

Presentación oral

Characteristics of Patients with Thyroid Cancer Evaluated by positron Emission Tomography in Imbanaco Medical Center

Pseudonym: PET

Vol. 6, supl. 1, p. 62

Presentación oral

Prevalencia del hipotiroidismo y caracterización clínica en gestantes del Eje Cafetero (Colombia), 2014-2017: estudio transversal

Seudónimo: Negroncho 1

Vol. 6, supl. 1, p. 63
Presentación oral

Proyecto genoma del cáncer en Colombia: análisis genético multinivel del cáncer papilar de tiroides (CPT) Seudónimo: GOMAVI2019

Vol. 6, supl. 1, p. 63-64

Póster

Ablación por radiofrecuencia de nódulos benignos de la tiroides: seguridad y eficacia hasta por 1 año en 25 pacientes

Hernández E, Forero OM, Herrera DF

Vol. 6, supl. 1, p. 96

Póster

Agranulocitosis inducida por metimazol: reporte de casos. Importancia de la detección temprana

Vargas, Silva MA, Coronel N, López N

Vol. 6, supl. 1, p. 96

Póster

Asociación de hipo- e hipertiroidismo con falla cardíaca

Barakat S, Ramírez A, Bernal W, Abreu A, Casanova M

Vol. 6, supl. 1, p. 97

Póster

Biopsia de aspiración con aguja fina en el diagnóstico de tiroiditis subaguda granulomatosa de De Quervain, serie de casos de 1996 a 2014

Montoya D, Vargas MA, Coronel N, Ramírez A, Vélez A, Aristizábal N, Torres JL, Palacio A, Botero JF

Vol. 6, supl. 1, p. 97

Póster

Bradicardia sinusal extrema en hipotiroidismo autoinmunitario, tiroiditis atrófica: reporte de caso Mejía HJ, Rodríguez L, Rangel KL, Rangel DA

Vol. 6, supl. 1, p. 97-98

Póster

Carcinoma de paratiroides: reporte de un caso

Quintero ML, Suárez L, Morales K, Peña J

Vol. 6, supl. 1, p. 98

Póster

Caso probable de metástasis cardíaca de carcinoma anaplásico de tiroides

Zea J, Román A, Londoño $S$

Vol. 6, supl. 1, p. 98-99

Póster

Determinación del volumen tiroideo medido por ecografía en Pereira, Risaralda

Arenas HM, López VJ

Vol. 6, supl. 1, p. 99

http://revistaendocrino.org/ 
Póster

Hiperparatiroidismo primario en mujeres

posmenopáusicas

Tascón BN

Vol. 6, supl. 1, p. 99

Póster

Hipertiroidismo gestacional transitorio: a propósito de dos casos

Vallejo S, Forero J, Oyola $\mathrm{K}$

Vol. 6, supl. 1, p. 100

Póster

Hipotiroidismo y micosis fungoide: una

asociación inusual

Saldarriaga LM, Bolaños OF

Vol. 6, supl. 1, p. 100

Póster

Plasmaféresis en tirotoxicosis y tormenta tiroidea como terapia puente para el manejo quirúrgico definitivo Aristizábal N, Coronel N, Vargas MA, Monsalve C, Torres JL, Arango CM, Prieto C, Builes CE

Vol. 6, supl. 1, p. 101

Póster

Tiroiditis supurativa con compromiso vascular en una paciente inmunocompetente (síndrome de Lemierre) Botero G, Méndez J, Benavides J, Tous R, Mejía M, Rojas W Vol. 6, supl. 1, p. 101

Póster

Tocilizumab en orbitopatía distiroidea

corticorresistente: serie de casos de un último recurso

Sánchez PE, Coy AF, Sierra A, Rojas W

Vol. 6, supl. 1, p. 101-102

Póster

Caracterización de pacientes con tirotoxicosis severa y tormenta tiroidea, Fundación Valle del Lili, 2011-2017

Martínez V, Arango LG, García L, Martínez JJ, Guzmán G

Vol. 6, supl. 1, p. 102

Póster

Utilidad de adicionar biopsia por congelación a la aspiración con aguja fina en pacientes con cáncer bien diferenciado de tiroides

Builes CE, Villa CA, Zapata NZ, Rojas N

Vol. 6, supl. 1, p. 102-104
Trabajos de investigación Misceláneos

Presentación oral

Consumo de azúcar y eventos cardiovasculares mayores. Revisión sistemática de la literatura

Seudónimo: azúcarcerebroycorazón

Vol. 6, supl. 1, p. 64

Presentación oral

Evaluación de la eficacia de dos terapias estrogénicas locales más un lubricante vaginal, para el control de los síntomas del síndrome genitourinario de la menopausia Seudónimo: Negroncho 2

Vol. 6, supl. 1, p. 65

Presentación oral

Evolución de los parámetros de espermograma en un instituto de fertilidad de Pereira, Risaralda en el período 2004-2018

Seudónimo: Espermatozoides

Vol. 6, supl. 1, p. 65-66

Presentación oral

Tibolona versus estrógenos conjugados de equino combinados con testosterona para el tratamiento del trastorno del deseo sexual hipoactivo en mujeres en climaterio. Un ensayo clínico aleatorizado Seudónimo: Negroncho 3

Vol. 6, supl. 1, p. 66

Póster

Amenorrea primaria e hiposmia: diagnóstico tardío del síndrome de Kallmann en una mujer

Ramírez A, Tutal L, Barakat S, Abreu A

Vol. 6, supl. 1, p. 104

Póster

Análisis de la dinámica de las variables morfológicas y de actividad física en estudiantes de Medicina. Estudio comparativo

Flórez V, Klinger H, Sánchez JJ, López AL

Vol. 6, supl. 1, p. 104-105

\section{Póster}

Cambios en la composición corporal y fuerza prensil en mujeres con programa de ejercicio bifásico con reacondicionamiento aeróbico y desadaptación muscular progresiva: presentación de una serie de casos Palacio JI, Polanco JP, Jaramillo A, Rosero RJ

Vol. 6, supl. 1, p. 105 
Póster

Caracterización de los cambios funcionales positivos con el entrenamiento en un grupo de atletismo de élite categoría máster en Valle del Cauca, Colombia

Revelo FJ, Tabares SA, Messa MC, Prado DG, Murcia A, López AL Vol. 6, supl. 1, p. 105-106

\section{Póster}

Caracterización del paciente sometido a flebotomía terapéutica y su relación con alteraciones endocrinológicas. Caso: banco de sangre nacional Collazos $Y E$

Vol. 6, supl. 1, p. 106

\section{Póster}

Efecto de los plaguicidas usados en cultivos de fresa, como posibles disruptores endocrinos: revisión bibliográfica

Tovar DG, Romero ED, Gutiérrez JC

Vol. 6, supl. 1, p. 106

\section{Póster}

Estruma ovárico maligno: ¿neoplasia primaria o metástasis? Reporte de un caso y revisión de la literatura Dueñas JP, Coronel N, Vargas MA, Aristizábal N, Torres JL, Botero JF, Echeverri LM, Martelo A

Vol. 6, supl. 1, p. 107

\section{Póster}

Hemocromatosis como causa de hipogonadismo hipogonadotrópico adquirido

Ramírez JJ, Vallejo S, Rodríguez MF, Marín HA

Vol. 6, supl. 1, p. 107

\section{Póster}

Realidades: género, experiencia y abordaje integral de los pacientes con incongruencia de género. Experiencia de los residentes del grupo interdisciplinario de incongruencia de género en el Hospital de San José Alarcón AM, Benítez JM, Gómez LM

Vol. 6, supl. 1, p. 107-108

\section{Póster}

La hipodinamia como factor de riesgo en estudiantes de medicina con normopeso de sexo femenino

Tabares SA, Messa MC, Revelo FJ, Prado DG, Murcia A, López AL Vol. 6, supl. 1, p. 108

\section{Póster}

Los fenotipos del síndrome de ovario poliquístico en mujeres de Colombia

Urdinola J, Martín R, Moreno B, Rodríguez N, Díaz IJ,

Hormaza MP, Cáceres LA

Vol. 6, supl. 1, p. 108-109
Póster

Motivos de interconsulta al servicio de endocrinología en un hospital universitario de tercer nivel de la ciudad de Pereira

González S, Vallejo S, Garzón V, Córdoba D

Vol. 6, supl. 1, p. 109

Póster

Perfil endocrinológico de la enfermedad de Huntington y posibles enfoques terapéuticos

Parga C, Padilla J, Fruto A, Tapia A, Perea S, Rosado C, Santodomingo $N$

Vol. 6, supl. 1, p. 109-110

Póster

Producción ectópica de la hormona liberadora de la hormona del crecimiento por un tumor neuroendocrino del hígado: respuesta a lanreotida

Castaño P, Román A, Builes CE

Vol. 6, supl. 1, p. 110

Póster

Relación entre el factor de crecimiento insulínico tipo 1 y las manifestaciones neurodegenerativas en la enfermedad de Huntington

Parga CH, Santodomingo NE, Tapia AD

Vol. 6, supl. 1, p. 110-111

Póster

Respuesta aguda del ejercicio resistido y concurrente en la glucemia posprandial de mujeres posmenopáusicas Rebolledo R, Sarmiento L, Becerra J, Ardila L

Vol. 6, supl. 1, p. 111

Póster

Uso e impacto de las redes sociales sobre las revistas biomédicas de endocrinología

Muñoz OM, Patiño D, Fernández DG, García AA, Gómez AM

Vol. 6, supl. 1, p. 111

Póster

Vipoma: causa poco frecuente de diarrea crónica secretora. Reporte de caso

Sánchez $S$, Torres $S$, Builes CA, Román $A$

Vol. 6, supl. 1, p. 112

Póster

Vitamina B12 sérica en un grupo de mujeres posmenopáusicas del departamento del Atlántico, Colombia, y su relación con variables bioquímicas y antropométricas

Sarmiento L, Becerra JE, Rebolledo R, Suárez A, Suárez H, Angarita J

Vol. 6, supl. 1, p. 112 